\title{
O Tratamento com Isoflavonas Mimetiza a Ação do Estradiol no Acúmulo de Gordura em Ratas Ovariectomizadas
}

\begin{abstract}
RESUMO
Objetivo: As isoflavonas (ISO) presentes na soja são consideradas fitoestrógenos. A administração de fitoestrógenos tem efeito benéfico nos distúrbios da pós-menopausa que são caracterizados pela suspensão da função ovariana com declínio da secreção de estrogênio e conseqüentes desajustes histomorfológicos e metabólicos. O objetivo do presente estudo foi investigar o efeito da suplementação com ISO sobre a espessura do endométrio uterino, o acúmulo de gordura tecidual, o colesterol HDL e a glicose plasmática de ratas ovariectomizadas (OVX). Métodos: Ratas Wistar com 60 dias de vida sofreram cirurgia bilateral para retirada dos ovários. Após o período de 8 dias de recuperação foram divididas em três grupos: falso operada (GC), OVX não-tratadas com ISO (GI) e as OVX suplementadas com ISO (GII). Foram retirados e pesados o útero, as gorduras uterinas e retroperitoneais. Também foram coletadas amostras de sangue para dosagem da concentração de HDL e glicose. Resultados: A OVX promoveu atrofia do endométrio, diminuição do peso do útero e diminuição do HDL. O tratamento com ISO promoveu diminuição dos estoques de gorduras uterina e retroperitoneal, aumento de HDL e redução da glicemia, porém não teve efeito uterotrófico. Conclusões: Os dados do presente estudo mostram que o tratamento com ISO promove redução da adiposidade, o que pode estar relacionado à redução da lipogênese e ao aumento da lipólise. (Arq Bras Endocrinol Metab 2008; 52/9:1489-1496)
\end{abstract}

Descritores: Isoflavonas; Fitoestrógenos; Ovariectomia; Menopausa; Adiposidade

\section{ABSTRACT}

Treatment with Isoflavones Replaces Estradiol Effect on the Tissue Fat Accumulation from Ovariectomized Rats.

Objective: Isoflavones (ISO) present in soybean are named phytoestrogens because they show estrogen effect. The use of isoflavones has beneficial effect in disturbance of post-menopause, which is characterized by ovarian function suppression. Decreasing of estrogen secretion and consequent morphologic and metabolic disarrangements are observed in female hormonal decline. The aim of present work was to investigate the effect of ISO on the fat accretion of uterin endometric tissue, and HDL and glucose blood concentration from ovariectomized rats (OVX). Methods: Female Wistar rats with 60 days-old were submitted a surgery to remove bilaterally the ovarium. After 8-day recovery period the animals were distributed into three groups: sham operate (GC); OVX ISO untreated (GI) and OVX supplemented with ISO (G II). Total uterus mass, uterus fat and retroperitoneal fat pad, were removed, washed and weighted. Samples of uterus were histological processed to measure endometrium thickness. Blood samples were also collected to analyze the concentration of HDL and glucose. The OVX caused endometric atrophy, decrease of uterus weight and HDL reduction. The treatment with ISO provoked decrease of uterin and retroperitoneal fat pad. HDL increase and glycemia reduction were also observed. However, there was no uterotrophic effect. Conclusions: ISO treatment causes decrease in tissue fat accretion from ovariectomized rats. (Arq Bras Endocrinol Metab 2008; 52/9:1489-1496)

Keywords: Isoflavones; Phytoestrogens; Ovariectomy; Menopause; Adiposity artigo original

\author{
ROSANA TORREZAN \\ RODRIGO M. GOMES \\ MARIA L. FERRARESE \\ FERNANDO BEN-HUR DE MELO \\ APARECIDA M. D. RAMOS \\ PAULO C. F. MATHIAS \\ DIONIZIA X. SCOMPARIN
}

Departamento de Ciências

Morfofisiológicas do Centro de

Ciências Biológicas da

Universidade Estadual de

Maringá (UEM) (RT, FBHM, AMDR);

Departamento de Biologia

Celular e Genética do Centro

de Ciências Biológicas da UEM

(RMG, PCFM, DXS);

Departamento de Bioquímica

do Centro de Ciências

Biológicas da UEM (MLF),

Maringá, PR, Brasil.

Recebido em 8/2/2008

Aceito em 17/10/2008 


\section{INTRODUÇÃO}

$\mathrm{s}$ isoflavonas (ISO) presentes na soja e seus derivados são denominados de fitoestrógenos por apresentarem semelhanças estrutural e funcional ao estradiol (1). Quando a soja e os seus produtos são consumidos, as ISO são hidrofilizadas no intestino delgado pela $\beta$-glicosidase intestinal. Esses fitoestrógenos são absorvidos ou fermentados pela microflora intestinal, e dão origem a seus metabólitos; daidzeína, gliciteína e genisteína, a qual é a mais abundante na soja e também a que apresenta maior ação estrogênica.

Uma parte das ISO absorvidas é transportada para o fígado, onde são removidas da circulação sanguínea por meio da veia porta, retornando ao intestino pela via biliar, podendo ser excretadas pelas fezes. Uma porcentagem, porém, chega até a circulação periférica, alcançando os tecidos (2).

Os fitoestrógenos se ligam aos receptores $\alpha$ e $\beta$ do estrógeno e desencadeiam as mesmas ações do estrógeno nos tecidos-alvo (3). Embora o efeito estrogênico desses fitoestrógenos seja muito fraco (4) eles podem exercer efeito agonístico e/ou antagonístico sobre os $\beta$-estrogênios endógenos, por competirem pelos mesmos receptores. O efeito biológico das ISO varia de acordo com a fase biológica da mulher. Na pré-menopausa, quando a concentração de hormônios circulantes é alta, o resultado é a fraca ação estrogênica ou antiestrogênica. Na pós-menopausa, quando a concentração do estrogênio endógeno diminui, em média $60 \%$, os receptores ficam mais disponíveis, favorecendo a ação estrogênica das ISO, que acabam compensando a deficiência do hormônio humano. Assim, além de proporcionarem melhora dos sintomas da menopausa, elas mantêm ação estrogênica positiva no metabolismo ósseo, no sistema nervoso central e no sistema cardiovascular (5-9).

Os estudos sobre a ação das ISO no útero de ratas ovariectomizadas (OVX) são bastante controversos. Geralmente feitos com a genisteína, a ISO de maior concentração na soja e seus derivados (10). A genisteína tem maior afinidade pelos receptores $\beta$-estrogênicos comparada com as outras ISO $(11,12)$.

Vários estudos mostram que os fitoestrógenos, causam crescimento uterino, em animais intactos e OVX $(12,13)$. Também foi observado que as ISO estimulam o crescimento de tumores responsivos ao estrogênio (14). A genisteína suplementada em ratas OVX $(750 \mathrm{mg} / \mathrm{kg}$ de peso corporal) apresenta efeito estrogênico no útero, na glândula mamária e no eixo hipotálamo-hipofisário, por diminuir a secreção de prolactina (15).

A menopausa é caracterizada pela suspensão irreversível da função ovariana, com declínio da secreção estrogênica. A falta desse hormônio leva a alterações no perfil lipídico, em particular, aumento das lipoproteínas de baixa densidade (LDL), redução das lipoproteínas de alta densidade (HDL) e aumento na deposição de gordura (16). Assim, a administração de fitoestrógenos tem efeito benéfico no perfil lipídico e no metabolismo hepático, na diminuição da deposição de tecido adiposo branco e na proteção contra doenças cardiovasculares na pós-menopausa $(2,17-20)$.

O tecido adiposo é responsivo ao estrogênio e expressa os receptores $\alpha$ e $\beta$ para este hormônio em humanos e roedores (21-24). O receptor $\alpha$ para $O$ estrogênio é o responsável pela modulação da lipogênese, no tecido adiposo (25), diminuindo a atividade da lipoproteína lipase (LPL), enzima que regula a captação de lipídios pelos adipócitos, sendo assim, a estimulação do receptor $\alpha$ provoca a redução do acúmulo de gordura (26). A administração de genisteína aumenta a expressão dos receptores para estrogênio, principalmente os do tipo $\alpha(15,27)$, o que reduz o aumento do tecido adiposo e do peso corporal de ratas OVX, mimetizando a ação do estradiol $(28,29)$.

A atuação dos isoflavonóides sobre o receptor $\beta$-estrogênico, presente no fígado, tem como conseqüência a melhora do perfil lipídico, possibilitado pelo incremento no número de receptores hepáticos de colesterol LDL. Dessa forma, o catabolismo de colesterol é favorecido (30). A estimulação dos receptores $\beta$-estrogênicos leva à inibição da lipase hepática, que está envolvida no metabolismo de colesterol HDL, ocasionando seu incremento (31). A isoflavona também estimula a $\beta$-oxidação dos ácidos graxos em várias espécies animais, incluindo o homem (32).

Considerando os comentários anteriores, a dieta suplementada com ISO tem sido utilizada no tratamento da síndrome metabólica e da obesidade. Trabalhos revelam efeitos benéficos das ISO sobre a tolerância à glicose na obesidade e no metabolismo hepático. Banz e cols. (33) mostraram que ratos $\mathrm{Zu}$ cker, geneticamente obesos, após o tratamento com ISO, apresentaram redução do peso corporal e do conteúdo de gordura, reduzindo o risco de lipotoxicidade hepática e, consequentemente, diminuindo o risco de esteatose (34). 
Conhecendo os efeitos dos isoflavonóides sobre o crescimento uterino, metabolismo lipídico e tecido adiposo, o objetivo deste estudo foi investigar o efeito da administração de ISO sobre a espessura do endométrio uterino e o acúmulo de gordura em ratas OVX.

\section{MÉTODOS}

\section{Animais e tratamento}

Ratas virgens com 60 dias de idade foram obtidas do Biotério Central da Universidade Estadual de Maringá e mantidas no Biotério Setorial do Departamento de Ciências Morfofisiológicas por oito dias antes do início dos experimentos, com ração e água à vontade, e sob ciclo fotoperiódico constante de 12 horas de claro e 12 horas de escuro (das 7 às 19), em temperatura de $22 \pm$ $2{ }^{\circ} \mathrm{C}$. Após a adaptação, o grupo foi submetido à ovariectomia bilateral e outro grupo foi falsamente operado. Após o período de recuperação, as ratas foram divididas em três grupos: grupo-controle falso-operado $(\mathrm{GC}, \mathrm{n}=6)$, grupo OVX não-suplementado com ISO (GI, $\mathrm{n}=6$ ) e grupo OVX suplementado com ISO (GII, $\mathrm{n}=10)$. Para efetuar a cirurgia de retirada dos ovários, as ratas foram anestesiadas com a mistura ketamina (3,0 $\mathrm{mg})$ e xilazina $(0,6 \mathrm{mg})$ para cada $100 \mathrm{~g}$ de peso corporal. A cirurgia foi realizada seguindo procedimentos previamente descritos por Zarrow e cols. (35), respeitando as normas do Colégio Brasileiro de Experimentação Animal (Cobea). As ISO, sob a forma de extrato, foram preparadas em suspensão com água, levando-se em consideração o fator de correção, conforme especificação da indústria farmacêutica (Pharma Nostra, Rio de Janeiro, Brasil), visando à administração real de 300 $\mathrm{mg} / \mathrm{kg}$ de massa corporal/por dia, de suspensão com teor de ISO de $41,56 \%$, dos quais $1,67 \%$ é daidzina; $2,05 \%$ genistina; $3,31 \%$ gliceteina; $3,35 \%$ glicetina; $7,93 \%$ daidizeina e $23,25 \%$ genisteína. As ISO foram administradas por gavagem, durante 21 dias consecutivos. Durante esse período, todos os animais receberam dieta comercial (Nuvital, Curitiba, Brasil), isenta de ISO, e água à vontade. Um grupo de ratas OVX recebeu a gavagem somente com água isenta de ISO. Após o período de suplementação com ISO, todos os animais foram sacrificados, os úteros e suas gorduras foram retirados, lavados com salina e pesados. Os fragmentos dos cornos uterinos foram isolados e processados com formalina e usados posteriormente para emblocamento em parafina. Os cortes foram preparados e corados com hematoxilina e eosina. Usando lente milimetrada foi aferida a espessura do endométrio uterino (36).

As gorduras retroperitoneais foram retiradas, lavadas em solução salina e pesadas. Também foram retiradas amostras de sangue para dosagem de colesterol HDL e da glicemia, no estado alimentado.

\section{Determinação da concentração de colesterol HDL}

O colesterol HDL foi determinado pelo método enzimático colorimétrico de colesterol-oxidase (Gold Analisa ${ }^{\circledR}$. Neste método, a colesterol-oxidase catalisa a oxidação do colesterol e produz peróxido de hidrogênio. Os resultados foram expressos em $\mathrm{mg} / \mathrm{dL}$.

\section{Determinação da glicemia}

A determinação dos níveis plasmáticos de glicose foi realizada em alíquotas de plasma, por meio da utilização de método enzimático colorimétrico da glicose-oxidase (Gold Analisa ${ }^{\circledR}$ ). A glicemia foi avaliada em animais alimentados. Os resultados foram expressos em $\mathrm{mg} / \mathrm{dL}$.

\section{Analise estatística}

Todos os grupos de animais foram estudados em paralelo. A comparação entre os diferentes grupos foi realizada empregando-se ANOVA. Quando as análises indicaram $\mathrm{F}$ significativo, as diferenças entre as médias foram testadas utilizando-se o teste de comparações múltiplas de Tukey-Kramer e o nível de significância adotado foi de $\mathrm{p}<0,05$.

\section{RESULTADOS}

\section{Histomorfologia e peso uterino}

A avaliação histomorfológica dos úteros (Figura 1) mostra que a OVX resultou redução de $55,01 \%$ na espessura do endométrio comparado ao grupo GC ( $\mathrm{p}<0,05)$, todavia, o tratamento com ISO não preveniu a atrofia induzida pela OVX (GII). A mesma redução foi observada para o peso dos úteros, $70,77 \%$ menor nas ratas OVX comparado às ratas falsamente operadas (Figura 2). A gavagem com água em ratas OVX não causou qualquer alteração comparada ao grupo de ratas OVX que não recebeu o tratamento com ISO (resultados não apresentados). 


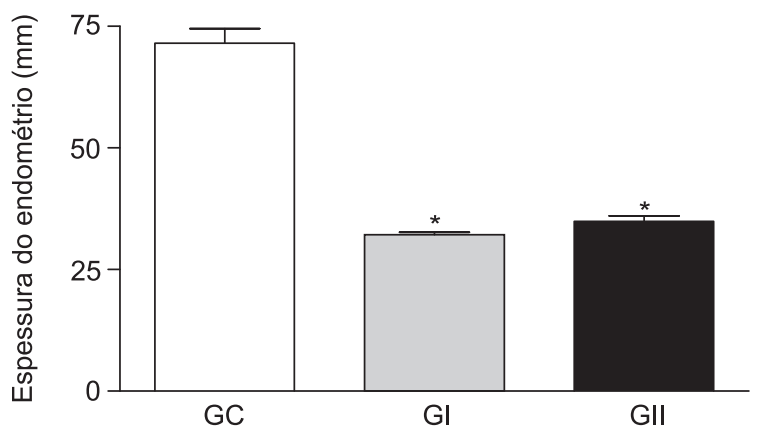

* Indica diferença significativa de p < 0,001 para os grupos Gl e Gll, comparado ao grupo GC.

Figura 1. Os dados representam a média \pm EPM da espessura do endométrio dos úteros de ratas-controle (GC), ratas OVX (GI) e de ratas OVX tratadas com ISO (GII).

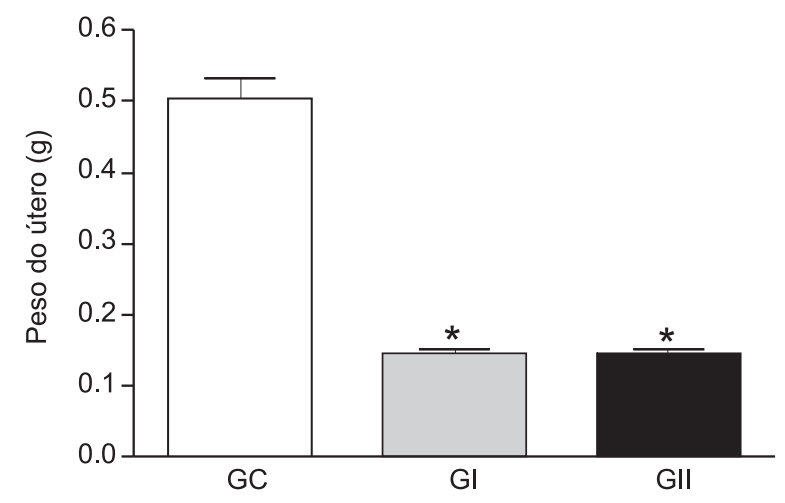

* Indica diferença significativa de p <0,001 para os grupos GI e Gll, comparado ao grupo GC.

Figura 2. Os dados representam a média \pm EPM do peso dos úteros de ratas-controle (GC), ratas OVX (GI) e de ratas OVX tratadas com ISO (GII).

A retirada dos ovários não promoveu alteração no peso da gordura peri-uterina comparadas ao grupocontrole, todavia, a administração de ISO promoveu redução de $28,74 \%$ no peso da gordura peri-uterina das ratas OVX p $<0,01$ (Figura 3).

\section{Gordura retroperitoneal}

A suplementação com ISO nas ratas OVX promoveu redução de $26,37 \%$ nos estoques de gordura retroperitoneal quando comparadas com o grupo GC, e $33,18 \%$ quando comparadas ao grupo GI $p<0,05$ (Figura 4).

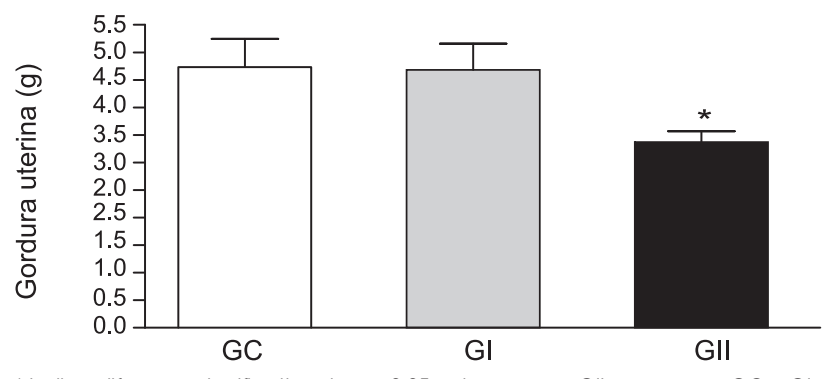

* Indica diferença significativa de $p<0,05$ entre o grupo Gll e os grupos GC e GI.

Figura 3. Efeito do tratamento com ISO sobre o peso da gordura uterina. Os dados representam a média \pm EPM.

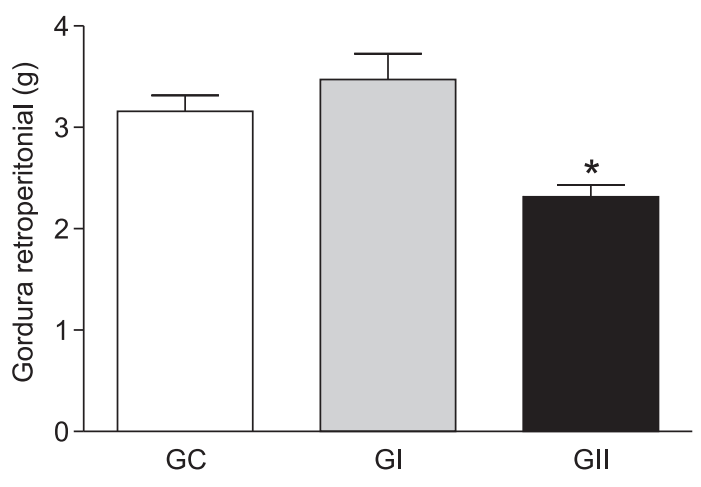

* Indica diferença de $p<0,05$ entre o grupo Gll e os grupos GC e Gl.

Figura 4. Efeito do tratamento com ISO sobre o peso da gordura retroperitoneal. Os dados representam a média \pm EPM.

\section{Colesterol HDL}

Os resultados sobre concentração plasmática de colesterol HDL mostram que a eliminação da ação do estrogênio, por meio da OVX, causou redução significativa de $66,24 \%$ nos níveis de HDL, entretanto, o tratamento com ISO restabeleceu a concentração de HDL no nível dos animais-controle, em que o grupo GII apresentou valor médio $175,83 \%$ maior que o grupo GI (Figura 5).

\section{Glicemia}

A glicemia no estado alimentado não diferiu entre os grupos não-tratados com ISO, GC e GI, porém os animais que receberam suplementação com ISO do grupo GII, tiveram redução na glicemia de $33,97 \%$, quando comparado aos animais do grupo GC e de $32,54 \%$, quando comparado ao grupo GI $(\mathrm{p}<0,05)$, como indica a Figura 6. 


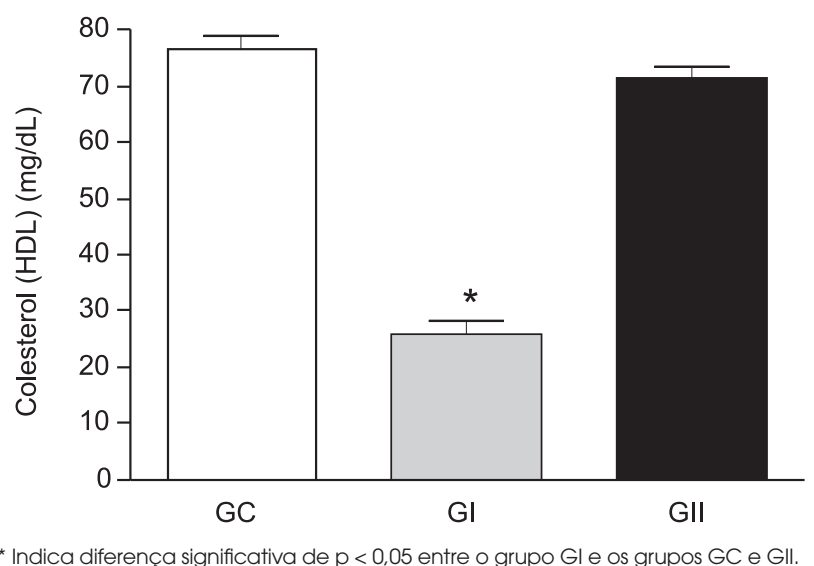

Figura 5. Efeito do tratamento com ISO na concentração plasmática de colesterol HDL. Os valores representam a média \pm EPM.

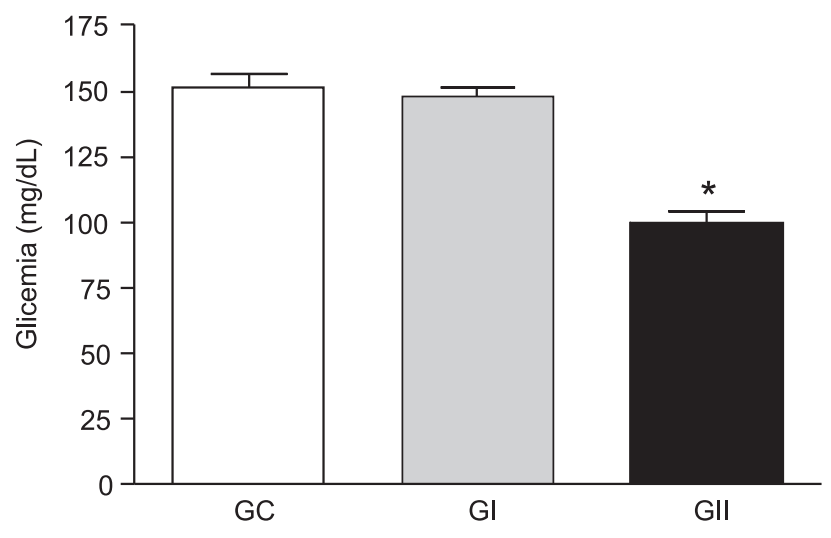

* Indica diferença significativa de p < 0,05 entre o grupo Gll e os grupos GC e Gl.

Figura 6. Efeito do tratamento com ISO na concentração plasmática de glicose. Os dados representam a média \pm EPM.

\section{DISCUSSÃo}

Os resultados histomorfológicos dos cornos uterinos e de pesos dos úteros do presente estudo deixam claro que o tratamento com ISO não teve ação uterotrófica sobre o endométrio das ratas OVX, considerando que as ISO não preveniram o efeito atrófico, causado pela ausência de estrogênio, em razão da ovariectomia. É importante ressaltar que o mesmo efeito foi obtido quando foram usadas doses de ISO maiores do que as usadas neste estudo(15).
Neste contexto, não obstante o conhecido efeito estrogênico das ISO, os resultados deste estudo e os de outros pesquisadores, indicam que, sobre o endométrio uterino, elas não apresentam qualquer efeito $(37,39)$. Tal fato poderia derivar da não-responsividade das ISO dos receptores estrogênicos, presentes no endométrio. Foram realizados alguns estudos a respeito da afinidade das ISO aos receptores $\alpha$ e $\beta$-estrogênicos; a genisteína tem maior afinidade por receptores $\beta$-estrogênicos, enquanto o estrogênio interage mais facilmente com os receptores $\alpha$-estrogênicos $(11,12,40)$. Cotroneo e cols. (41) não constataram efeito da genisteína na dose de $250 \mathrm{mg} / \mathrm{kg}$ sobre os receptores $\alpha$-estrogênicos. O principal receptor estrogênico do útero é o subtipo $\alpha$, para o qual a genisteína tem baixa afinidade $(11,40,27)$. Estas observações podem explicar a ausência de efeito uterotrófico das ISO, encontrado pelo grupo do presente estudo e por outros pesquisadores em ratas OVX. Reforçando estes achados, alguns estudos têm mostrado que as ISO apresentam ação seletiva, isto é, exibem atividade estrogênica em alguns tecidos e antiestrogênica em outros (42).

Os resultados do presente estudo mostram que o tratamento das ratas OVX com ISO promoveu redução significativa no acúmulo das gorduras peri-uterina e retroperitonial em comparação às ratas operadas e falsasoperadas que não receberam ISO. Esses resultados são semelhantes a dados da literatura, que relacionam o uso de ISO com menor acúmulo de tecido adiposo branco, indicando efeito antilipogênico (43-46).

A administração de estrogênio diminui o número e o tamanho de adipócitos, enquanto sua deficiência resulta aumento deles (47). Neste contexto, vários trabalhos têm sido realizados para investigar os efeitos benéficos dos fitoestrógenos sobre a adiposidade (15, 27-29).

A genisteína tem efeito direto sobre o metabolismo de lipídios no fígado e no tecido adiposo. As ISO, sobretudo a genisteína, aumentam a lipólise em adipócitos isolados de ratos, e diminuem a lipogênese no tecido adiposo branco de ratas OVX (48). A incubação de adipócitos de ratos com genisteína resultou inibição da conversão da glicose em lipídios na ausência ou na presença de insulina, com subseqüente redução da lipogênese $(49,50)$. Outra ação da genisteína é a diminuição da expressão do RNA mensageiro da LPL no tecido adiposo, com conseqüente diminuição na deposição de gordura (44). 
Todos os dados referentes à ação antilipogênica das ISO, descritos anteriormente, sugerem mecanismo de redução no acúmulo de gordura no tecido adiposo branco exercido pelo tratamento com ISO.

No presente estudo, ratas Wistar submetidas a OVX constituem modelo animal para os estudos das alterações pós-menopáusicas, como mudanças no perfil lipídico. A análise dos níveis sanguíneos do colesterol HDL mostrou que este foi menor no grupo OVX do que no grupo OVX tratado com ISO e no grupo-controle. Estes resultados indicam que as ISO apresentam efeitos positivos no perfil lipídico de ratas OVX. Esta observação é consistente com os relatos de vários pesquisadores, que também utilizaram como modelo experimental ratas OVX tratadas com ISO $(48,51,52)$.

Não está esclarecido como as ISO agem para restabelecer as alterações lipídicas causadas pela menopausa. Contudo, tem sido proposto que a atuação das ISO, sobre receptores $\beta$-estrogênicos presentes no fígado, melhora o perfil lipídico por meio do incremento do número de receptores hepáticos do colesterol LDL, o que favorece o catabolismo de colesterol (17). A estimulação dos receptores $\beta$-estrogênicos levaria à inibição da lípase hepática, implicada no metabolismo do colesterol HDL, ocasionando seu incremento $(31,48)$.

Os resultados do presente estudo indicam que as ratas tratadas com ISO apresentaram queda significativa na glicemia durante o estado alimentado. Reforçando os resultados, Lavigne e cols. (53) mostraram que ratos alimentados com soja apresentam menor área sob a curva da glicose e da insulina plasmáticas durante o teste de tolerância à glicose intravenoso. Cheng e cols. (54) relataram que mulheres na pós-menopausa, tratadas com ISO apresentaram diminuição da glicemia de jejum e dos níveis de insulina. No entanto, ainda não são claros os mecanismos pelos quais as ISO causam o efeito hipoglicêmico em ratas OVX.

Em síntese, os resultados do presente estudo mostram que o tratamento com pequena dose de ISO reduz o acúmulo de gordura em ratas OVX e que este efeito pode estar relacionado com a redução da lipogênese e o aumento da lipólise.

Agradecimentos: Fundação Araucária, Coordenação de Aperfeiçoamento de Pessoal de Nível Superior (Capes) e Conselho Nacional de Desenvolvimento Científico e Tecnológico (CNPq) pelo apoio financeiro e a Pharma Nostra que, gentilmente, cedeu a mistura de ISO. Não há conflitos de interesse por parte dos autores, dos órgãos financiadores e da empresa colaboradora que possam interferir na publicação dos resultados contidos neste trabalho.

\section{REFERÊNCIAS}

1. Knight DC, Eden JA. A review of the clinical effects of phytoestrogens. Obstet Gynecol. 1996; 87:897-904.

2. Setchell KDR, Cassidy A. Dietary isoflavonas: biological effects and relevance to human heall. J Nutr. 1999;129(3):758 S-67.

3. Farmakalidis E, Hathcock JN, Murphy PA. Oestrogenic potency of genistin and daidzin in mice. Food Chem Toxicol. 1985; 23:741-5.

4. Setchell KDR, Zimmer-Nechemias L, Cai J, Heubi JE. Exposure of infants to phyto-oestrogens from soy-based infant formula. Lancet. 1997;350:23-7.

5. Clarkson TB, Anthony MS, Williams JK, Honore EK, Cline JM. The potential of soybean phytoestrogens for postmenopausal hormone replacement therapy. Proc Soc Exp Biol Med. 1998; 217:365-8.

6. Gennari C, Adami S, Agnusdei D. Effect of chronic treatment with ipriflavone in postmenopausal women with low bone mass. Calcif Tissue Int. 1997;61(Suppl 1):S19-22.

7. Arjmandi BH, Alekel L, Hollis BW. Dietary soybean protein prevents bone loss in an ovariectomized rat model of osteoporosis. J Nutr. 1996;126:161-7.

8. Lephart ED, Setchell KD, Handa RJ, Lund TD. Behavioral effects of endocrine-disrupting substances: phytoestrogens. ILAR J. 2004;45(4):443-54.

9. Bu L, Setchell K, Lephart E. Influences of dietary soy isoflavones on metabolism but not noriception and stress hormone responses in ovariectomized female rats. Reprod Biol Endocrinol. 2005;(3):58-65.

10. Reinli K, Block G. Phytoestrogen content of foods - a compendium of literature values. Nutr Cancer. 1996;26:123-48.

11. Kuiper GJM, Lemmen JG, Carlsson B, Corton JC, Safe SH, Van Der Saag PT, et al. Interaction of estrogenic chemicals and phytoestrogens with estrogen receptor $\beta$. Endocrinology. 1998;139:4252-63.

12. Makella S, Sabolinem H, Aavik E, Myllatviniemi M, Strauss L, Taskinen $E$, et al. Differentiation between vasculoprotective and uterotrophic effects of ligands with different binding affinities to estrogen receptor $\alpha$ and $\beta$. Environ Health. 1999;102: 572-8.

13. Mosquette R, Simões JM, Simões RS, Rondon AS, Bacarat EC, Simões MJ. Efeito do estrogênio eqüino e da isoflavona no útero de ratas adultas castradas sobre a atividade proliferativa (PCNA). Arq Inst Biol. 2005;72 Suppl 2:449-52.

14. Diel P, Smolnikar K, Schulz T, Laudenbach-Leschowski U, Michna H, Vollmer G. Phytoestrogens and carcinogenesis-differencitial effects of genistein in experimental model of normal of normal and malignant rat endometrium. Hum Reprod. 2001;16:997-1006.

15. Santell RC, Chang YC, Nair MG, Helferich WG. Dietary genistein eseerts estrogenic effects upon the uterus, mammary gland and the hypothalamic/pituitary aseis in rats. J Nutr. 1997;127:263-9.

16. Wong WW, Smith EO, Stuff JE. Cholesterol-lowering effect of soy protein in nomocholesterolemic and hypercholesterolemic men. Am J Clin Nutr. 1998;Suppl 1385:S-9. 
17. Sirtori CR, Gianazza E, Manzoni C, Lovati MR, Murphy PA. Role of isoflavones in the cholesterol reduction by soybean proteins in the clinic. Am J Clin Nutr. 1997;65:166-7.

18. Sirtori CR. Risks and benefits of soy phytoestrogens in cardiovascular diseases, cancer, climacteric symptoms and osteoporosis. Drug Safety. 2001;24:665-82.

19. Blair RM, Appt SE, Bennetau-Pelissero C, Clarkson TB, Anthony MS, Lamothe $V$, et al. Dietary soy and soy isoflavones have gender-specific effects on plasma lipids and isoflavones in golden Syrian F1B hybrid hamsters. J Nutr. 2001;132:3585-91.

20. Dewell A, Hollenbeck PLW, Hollenbeck CB. A critical evaluation of the role of soy protein and isoflavone supplementation in the control of plasma cholesterol concentrations. J Clin Endocrinol Metab. 2006;91(3):772-80.

21. Cooke TS, Naaz A. Role of estrogens in adipocyte development and function. Exp Biol Med. 2004;229(11):1127-35.

22. Pedersen SB, Hansen PS, Lund S, Andersen PH, Odgaard A, Richelsen $B$. Identification of oestrogen receptors and oestrogen receptor mRNA in human adipose tissue. Eur J Clin Invest. 1996;26:262-9.

23. Mueller SO, Clark JA, Myers PH, Korach KS. Mammary gland development in adult mice requires epithelial and stromal estrogen receptor $\alpha$. Endocrinology. 2002;143:2357-65.

24. Naaz A, Zakroczymski M, Heine P, Taylor J, Saunders P, Lubahn $D$, et al. Effect of ovariectomy on adipose tissue of mice in the absence of estrogen receptor $\alpha(E R \alpha)$ : a potential role for estrogen receptor $\beta$ (ER $\beta$ ). Horm Metab Res. 2001;34:758-63.

25. Heine PA, Taylor JA, Iwamoto GA, Lubahn DB, Cooke PS. Increased adipose tissue in male and female estrogen receptor- $\alpha$ knockout mice. Proc Natl Acad Sci U S A. 2000;97:12729-34.

26. Gentry RT, Wade GN. Sex differences in sensitivity of food intake, body weight, and running-wheel activity to ovarian steroids in rats. J Comp Physiol Psychol. 1976;90(8):747-54.

27. Hughes CL, Lui G, Beall S, Foster WG, Davis V. Effects of genisteins or soy milk during late gestation and location on adult uterine organization in the rat. Exp Biol Med. 2004;229:108-17.

28. Mohamed MK, Abdel-Rahman AA. Effect of long-term ovariectomy and estrogen replacement on the expression of estrogen receptor gene in female rats. Eur $\mathrm{J}$ Endocrinol. 2000;142:307-14.

29. Hamosh M, Hamosh P. The effect of estrogen on the lipoprotein lipase activity of rat adipose tissue. J Clin Invest. 1975; 55:1132-5.

30. Potter SM, Pertile J, Berber-Jimenez MD. Soy protein concentrate and isolated soy protein similarly lower blood serum cholesterol but differently affect thyroid hormones in hamsters. J Nutrition. 1996;126(8):2007-11.

31. Anderson JW, Johnstone BM, Cook Newell ME. Meta-analysis of the effects of soybean protein intake on serum lipids. $\mathrm{N}$ Engl J Med. 1995;333:276-82.

32. Volgarev MN, Vysotosky VG, Meshcheryakova VA, Yatsyshina TA, Steinke FH. Evaluation of isolated soy protein foods in weight reduction with obese hypercholesterolemic and normocholesterolemic obese individuals. Nutr Rep Int. 1989;36: 61-72.

33. Banz WJ, Jeremy D, Muhammad JI. Gene expression and adiposity are modified by soy protein in male Zucker diabetic fatty rats. Obes Res. 2004;12:1907-13.

34. Tovar AR, Torre-Villarlvazo I, Ochoa M, Elias AL, Ortiz V, Aguilar-Salinas CA, et al. Soy protein reduces hepatic lipotoxicity in hyperinsulinemic obese Zucker fa/fa rats. J Lipid Res. 2005;46(9):1823-32.
35. Zarrow MX, Yachim JM, Mccarthy JL, Sanbom RC. Experimental endocrinology a sourcebook of basic techniques. New York: Academic Press; 1964. p. 39-40.

36. Diel P, Laudenbach-Leschowsky U, Friedel A, Voss A, Roussel J. Pulsed estradiol exposure has a limited ability to induce uterine proliferation in ovariectomised female Wistar rats. Mol Cell Endocrinol. 2005;230:7-15.

37. Zierau O, Geis RB, Tischer S, Schwab P, Netz P, Vollmer G. Uterine effects of the phytoestrogen 6-(1,1-dimethylallyl) naringenin in rats. Planta Med. 2004;70(7):590-3.

38. Gallo D, Zannoni GF, Apollono P, Martinelli E, Ferlini C, Passetti $G$, et al. Characterization of the pharmacologic profile of a standardized rat extract in the ovariectomized rat model of menopause: effects on bone, uterus, and lipid profile. Menopause. 2005;12(5):589-600.

39. Nakai M, Cook L, Pyter LM, Black M, Sibona J, Turner RT, et al. Dietary soy protein and isoflavone have no significant effect on bone and a potentially negative effect on the uterus of sexually mature intact Sprangue-Dawley female rats. Menopause. $2005 ; 12(3): 291-8$.

40. Lee GS, Choi KC, Kim HJ, Jeung EB. Effect of genistein as a selective estrogen receptor beta agonist on the expression of cabindim-D in the uterus of immature rats. Toxicol Sci. 2004;82(2):451-7.

41. Cotroneo MS, Fritz WA, Lamartiniere CA. Dynamic profiling of estrogen receptor and epidermal growth factor signaling in the uteri of genistein- and estrogen-treated rats. Food Chem Toxicol. 2005;43(5):637-45.

42. Bahr JM, Nakai M, Rivera A, Walsh J, Evans GL, Lotinun S, et al. Dietary soy protein and isoflavones: minimal beneficial effects on bone and no effect on the reproductive tract of sexually mature ovariectomized Sprague-Dawley rats. Menopause. 2005;12(2):165-73.

43. Nagasawa A, Fukui K, Funahashi T, Maeda N, Shimomura I, Kihara S, et al. Effects of soy protein diet on the expression of adipose genes and plasma adiponectin. Horm Metab Res. 2002;34(11-2):635-9.

44. Naaz A, Yeliayi S, Zakroczymski MA, Burrick D, Dorge DR, Lubahn DB, et al. The soy isoflavone genistein decreases adipose deposition im mice. Endocrinology. 2003;144:3315-20.

45. Manzoni MS, Rossi EA, Carlos IZ, Vendramini RC, Duarte AC, Damasco AR. Fermented soy product supplemented with isoflavones affected fat depots in juvenile rats. Nutrition. 2005;21(10):1018-24.

46. Kim HK, Nelson-Dooley MA, Della-Fera JY, Jang W, Zhang J, Duan J, et al. Genistein decreases food intake, body weight, and fat pad weight and cause adipose tissue apoptosis in ovariectomized femal mice. J Nutr. 2006;136(2):409-14.

47. Cooke PS, Heine PA, Taylor JA, Lubahn DB. The role of estrogen and estrogen receptor-alpha in male adipose tissue. Mol Cell Endocrinol. 2001;178(1-2):147-54.

48. Nogowski L, Mackowiak P, Kandulska K, Szkudelski T, Nowak KW. Genistein-induced changes in lipid metabolism of ovariectomized rats. Ann Nutr Metab. 1998;42(6):360-6.

49. Persaud SJ, Harris TE, Burns CJ, Jones PM. Tyrosine kinases play a permissive role in glucose-induced insulin secretion from adult rat islets. J Mol Endocrinol. 1999;22(1):19-28.

50. Szkudelska K, Nogowski L, Szkudelsk T. Genistein affects lipogenis and lipolysis in isolated rat adipocytes. J Esteroid Biochem Mol Biol. 2000;75(4-5): 265-71. 
51. Uesugi T, Toda T, Tsuji K, Ishida H. Comparative study on reduction of bone loss and lipid metabolism abnormality in ovariectomized rats by soy isoflavones, daidzin, genistin, and glycitin. Biol Pharm Bull. 2001;24:368-72.

52. Wang JF, Niu JZ, Zhang C, Liu LQ, Gao BH. Effects of soy extract on lipid metabolism in ovariectomized rats. Zhongguo Zhong Yao Za Zhi. 2002;27(4):285-8.

53. Lavigne C, Marette A, Jacques H. Cod and soy proteins compared with casein improve glucose tolerance and insulin sensitivity in rats. Am J Fisiol Endocrinol Metab. 2000;278(3): E491-500.
54. Cheng SY, Shaw NS, Tsai KS, Chen CY. The hypoglycemic effects of soy isoflavones on postmenopausal women. J Womens Health. 2004;3(10):1080-6.

\section{Endereço para correspondência:}

Dionizia Xavier Scomparin

Universidade Estadual de Maringá

Dep. de Biologia Celular e Genética, bloco H-67, sala 19

Av. Colombo, 5790

87020-900 Maringá PR

E-mail: scomparindio@hotmail.com 